\title{
Ascending Colon Neuroendocrine Tumor
} G1

National Cancer Institute

\section{Source}

National Cancer Institute. Ascending Colon Neuroendocrine Tumor G1. NCI Thesaurus.

Code C6427.

A well differentiated, low grade neuroendocrine tumor (carcinoid tumor) that arises from the ascending colon. The mitotic count is less than 2 per 10 HPF and/or the Ki67 index is equal to or less than 2 percent. 